\title{
Effects of radiotherapy on wound healing
}

Keywords: radiotherapy, wound healing, tissue injury

Radiotherapy is widely used to treat for cancer. This therapy aims to kill cancer cells while sparing the surrounding tissue where possible. Radiotherapy can impair the normal healing process in any phase of tissue repair. This review discusses the acute and late effects of radiotherapy and relates them to wound healing.

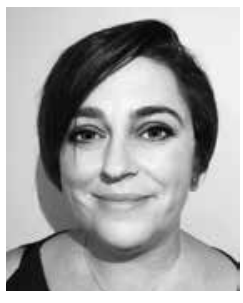

Pauline J Robinson RN, Year 1 Student Masters in Wound Healing and Tissue Repair Centre for Medical Education, School of Medicine, Cardiff University Wales, UK

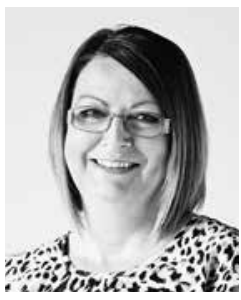

Samantha L Holloway MSc, Reader, Programme Director. Masters in Wound Healing and Tissue Repair Centre for Medical Education, School of Medicine Cardiff University, Wales, UK

\section{Aim}

To present a narrative review of the current evidence related to the effects of radiotherapy on normal wound healing.

\section{Findings}

Fractionated radiotherapy treatment induces a repetitive cycle of inflammation. The acute effects of radiotherapy are mostly transient and are resolved within 4 weeks of the last dose, which correlates with the normal pathogenesis of skin regeneration. Two main theories exist with regards to the late effects of radiotherapy and the development of a chronic wound. One hypothesis is that there is a hypoxic-hypocellular-hypovascular effect. Other authors have argued that damage occurs as a result of changes to the microvasculature, in addition to the depletion of parenchymal and stromal cells. Conclusion: Recent evidence indicates that advances in treatment modalities, for example, the use of proton therapy and intensity-modulated radiotherapy, can positively influence patient outcomes in relation to tissue injury. Further advancements in our understanding of radiotherapy and its effects may lead to an improvement in the type of treatment modalities available, thereby helping prevent tissue injury altogether. Clinicians need to appreciate that healing of a radiation wound may not be realistic. Symptom-relieving treatment is of paramount importance to address the deleterious consequences of a radiotherapy related wound.
Clinicians also need to be cognisant of the need to maintain the patient's self-esteem and maximise their quality of life.

\section{Key Messages}

This review explores the existing evidence related to the effects of radiotherapy on normal wound healing. The main observations from the studies examined indicate that ionising radiation damages cellular molecules primarily through strand breaks of deoxyribonucleic acid (DNA) or by the production of free radicals and reactive oxygen species, affecting both normal and tumour cells. Radiotherapy can impair the normal healing process in any phase of tissue repair as a result of cellular depletion, alteration in the extracellular matrix (ECM), and impairment of the microvasculature with subsequent tissue hypoxia. The acute effects of radiotherapy on the tissues range in severity from erythema to dry desquamation and hyperpigmentation. In the months or years following radiotherapy, tissue breakdown may occur leading to skin atrophy, dryness, telangiectasia, dyschromia, and dyspigmentation.

\section{INTRODUCTION}

Radiotherapy has been used as a treatment modality for over a century, and currently, it is used as a standalone cancer treatment or as an adjunct to surgery or chemotherapy. ${ }^{1}$ The two main types of radiotherapy are as follows: electromagnetic radiation with $\mathrm{x}$-rays and gamma rays and particulate radiation using electrons, neutrons, and protons. ${ }^{2}$ Radiation is delivered through multiple modalities, with external beam being the most commonly used modality. ${ }^{1,3}$ The treatment modality depends on the anatomical location, size, and type of
Correspondence: hollowaysl1@cf.ac.uk

Conflicts of interest: None 
the tumour ${ }^{2}$. The aim of the treatment is to kill the tumour cells while sparing the unaffected surrounding tissue. ${ }^{1,4}$ Nevertheless, radiotherapy causes inadvertent damage to adjacent normal tissues in up to $60 \%$ of surgical patients, especially to rapidly dividing tissues, such as skin, which are more radiosensitive. ${ }^{3,5}$ Ionising radiation acts either directly by damaging cellular molecules through energy transfer-primarily through strand breaks of deoxyribonucleic acid (DNA) or indirectly through the production of free radicals and reactive oxygen species-affecting both normal and tumour cells. ${ }^{3,6}$ Single-strand DNA breaks are thought to cause sub-lethal cellular damage which can be repaired, whereas double-strand DNA breaks are believed to cause lethal cellular damage. ${ }^{1}$

\section{NORMAL WOUND HEALING}

The normal tissue-repair process involves a well-orchestrated overlapping sequence of cellular and biochemical events, including inflammation, proliferation, and remodelling. ${ }^{7-8}$ A number of intrinsic and extrinsic factors, such as poor nutrition, diabetes, infection, and the presence of a biofilm, can alter this finely tuned process. ${ }^{9}$ Radiotherapy is an extrinsic factor that can impair or modify this well-regulated and coordinated sequence of events, and it can result in the formation of a chronic or non-healing wound that fails to proceed through the normal sequence of tissue repair. ${ }^{4,10}$ Radiotherapy instigates inflammatory cell recruitment, with skin damage occurring immediately after the initial and subsequent doses. ${ }^{6}$

Radiotherapy can impair the normal healing process in any phase of tissue repair as a result of cellular depletion, alteration in the extracellular matrix (ECM), and impairment of the microvasculature with subsequent tissue hypoxia. ${ }^{3}$ Complications are contingent on the total dose, dose per fraction, treatment modality and particles used, intervals between fractions, and quantity of tissue that receives a high dose. ${ }^{1-2}$ The effects of radiotherapy on tissue repair can be classified as acute and chronic. ${ }^{5}$

\section{ACUTE EFFECTS OF RADIOTHERAPY}

The acute effects of radiotherapy are observed in approximately $95 \%$ of patients. ${ }^{11}$ They range in severity from erythema to dry desquamation, hyperpigmentation, and hair loss. ${ }^{1,5}$ Acute effects usually appear within $1-4$ weeks of the initial treatment, and they can last for 2-4 weeks following the final treatment. ${ }^{1,11}$ A surge of free radicals modifies the proteins, lipids, carbohydrates, complex molecules, and DNA after each subsequent exposure, resulting in a cycle of inflammatory cell recruitment and tissue injury that inhibits tissue repair. ${ }^{12-13} \mathrm{~A}$ proportion of basal keratinocytes are destroyed following the first and subsequent fractionated doses of radiotherapy, and the residual cells become cornified, shedding more rapidly; consequently, the prolif- eration and differentiation of cells and the self-renewal of the epidermis are impaired.${ }^{11}$ The residual keratinocytes continue to proliferate, but they are destroyed by subsequent doses of radiation. ${ }^{13}$ Furthermore, the cytokines that are present during the inflammatory phase of tissue repair, for example, transforming growth factor beta (TGFĐ), vascular endothelial growth factor (VEGF), tumour necrosis factor alpha (TNFĐ), and interferon gamma (IFN-Đ), as well as the pro-inflammatory cytokines interleukin-1 and interleukin-8, are overexpressed. ${ }^{13}$ This prolongs the inflammatory phase of tissue repair and generates a buildup of the ECM, leading to fibrosis. 3,14

Liu et al. used a murine model and local soft x-ray irradiation to examine the cellular effects of irradiation on tissue repair. ${ }^{15}$ They suggested that the tissue-repair ability is dose dependent. Furthermore, they declared that all phases of wound healing are impaired by irradiation and attributed this impairment to inactive cell proliferation, apoptosis, and arrested cell cycle. They found this resulted in reduced inflammation, thinner granulation, and delayed re-epithelialisation.

\section{LATE EFFECTS OF RADIOTHERAPY}

In the months or years following radiotherapy, tissue breakdown may occur as a consequence of trauma, surgery, or infection, leading to the formation of chronic hard-toheal wounds. ${ }^{4}$ In addition, skin atrophy, dryness, telangiectasia, dyschromia, and dyspigmentation may occur. ${ }^{4} \mathrm{~A}$ frequent late effect of radiotherapy is fibrosis, which can lead to functional loss, body image issues, and psychosocial issues which can affect an individual's self-esteem and their quality of life. ${ }^{1}$ Keskikuru et al. suggested that fractionated radiotherapy is linked to a reduction in the severity of fibrosis. ${ }^{16}$

Based on an in vitro study of the pathogenesis of osteoradionecrosis, Marx (1983) proposed that the late effects of radiation, resulted in hypoxic-hypocellular-hypovascular tissue, which resulted in tissue breakdown, and as a consequence a chronic non-healing wound. ${ }^{17}$ This theory is now widely accepted ${ }^{18}$ and challenged the previous propositions of Meyer (1970) who suggested that that radiotherapy-induced tissue injury is caused by radiation, trauma, and infection. ${ }^{19}$ Dion et al. (1990) undertook a pilot study of the treatment effects of pentoxifylline on 12 patients with late radiation necrosis of soft tissues. ${ }^{20}$ Although the authors supported the concept of impaired healing due to vascular injury, they suggested that the late effects of radiotherapy resulted from the depletion of parenchymal and stromal cells. In addition, they reported that $87 \%$ of the patients treated with pentoxifylline exhibited complete wound closure with a median reduction 
in healing time of 9 weeks, suggesting that pentoxifylline could be therapeutically beneficial for healing irradiated wounds. Pareek et al. developed a protocol for a Cochrane systematic review to examine the individual and combined effects of pentoxifylline and vitamin $\mathrm{E}$ for preventing and treating the side effects of radiation therapy and concomitant chemoradiotherapy, the results of which are yet to be published. ${ }^{21}$ More recently, Famoso et al. examined patient compliance with pentoxifylline and vitamin $\mathrm{E}$ treatment after breast radiation in 90 patients and found a poor compliance rate in 33 of 87 patients (38\%); the main side effect was related to nausea. ${ }^{22}$

Illsley et al. examined the effect of irradiation on collagen production by fibroblasts cultured from the skin of patients undergoing radiotherapy. ${ }^{23}$ The authors found that collagen production was elevated in cells cultured from irradiated skin compared to that in cells cultured from un-irradiated skin $(\mathrm{p}=0.016)$. The addition of TGFĐ1 to un-irradiated skin fibroblasts increased the rate of collagen production, but the same effect was not observed with irradiated skin. In addition, it was observed that the cells from irradiated skin were larger than the control cells. They proposed that elevated collagen synthesis contributed to the development of fibrosis, and irradiation induced an altered fibroblast phenotype.

Goessler et al. studied tissue samples taken from three patients with squamous-cell carcinoma treated with postoperative radiotherapy. ${ }^{24}$ The patients presented severe non-healing dermal wounds in the neck area, which was previously irradiated. Histological analysis identified that the keratinocytes in the irradiated tissue failed to proliferate compared to the control. Gene expressions of cytokeratin 1 and 10 were significantly reduced in the irradiated tissue, whereas gene expressions of cytokeratin 5 and 14 were significantly elevated. Furthermore, transforming growth factor alpha, TGFĐ2, TGFĐ3, keratinocyte growth factor, hepatocyte growth factor (HGF), and VEGF expression were reduced significantly. The gene expressions of fibroblast growth factors 1 and 2 and HGF were reduced marginally. Furthermore, metalloproteinases (MMP) 7 , 12 , and 13 were significantly elevated in the irradiated keratinocytes and fibroblasts, and the gene expression of MMP2 was elevated marginally. These findings suggest that damage to the keratinocytes during the inflammatory phase impairs angiogenesis in the proliferative phase of tissue repair. Notably, these results pertaining to the acute effects of radiotherapy have been obtained with relatively small numbers of patients with non-healing chronic wounds, which makes it difficult to judge the significance of these results.

\section{WOUND TENSILE STRENGTH}

In normal wound healing, fibroblasts are attracted to the wound predominantly by platelet-derived growth factor and TGFĐ during the proliferative phase of tissue repair. ${ }^{8}$ The fibroblasts then proliferate, producing fibronectin, hyaluronan, collagen, and proteoglycans, which facilitate remodelling of the ECM. ${ }^{8}$ Type III Collagen, which is predominant during proliferation, is then synthesised by the fibroblasts. During the remodelling phase of healing, Type III collagen is replaced with Type I collagen, and the fibres are then re-arranged and cross-linked. ${ }^{7-8}$ This process imparts tensile strength to the wounded tissue. Notably, the wounded tissue never regains its original strength, but it does recover approximately $80 \%$ of the original strength at 12 weeks (Broughton et al. 2006). ${ }^{25}$

Schaffer et al. investigated the tensile strength of irradiated wounds. ${ }^{26}$ They established that impaired healing was associated with diminished nitric oxide (NO) synthesis and inducible NO synthase expression $(\mathrm{p}=0.01)$ and found upregulation of cell-signalling cytokine TNF- $Ð$ and proinflammatory cytokine IFN-Đ. Furthermore, they found diminished levels of $\mathrm{NO}$ synthesis and collagen deposition by fibroblasts $(p=0.01)$. In vitro irradiation of fibroblasts taken from non-irradiated rats led to reduced production of both NO and collagen ( $\mathrm{p}=0.01)$. This finding suggests that an imbalance between promoting and inhibiting factors impairs the healing of irradiated wounds.

Ozbek et al. investigated the effects of preoperative conventional and hyper-fractionated radiotherapy regimes on wound healing and wound tensile strength in rats. ${ }^{27}$ The rats were randomized into three groups, with each group comprising 30 rats; sham irradiated; conventional radiotherapy with 20 daily fractions of $200 \mathrm{cGy}$, resulting in a total dose of $4000 \mathrm{cGy}$; and hyper-fractionated radiotherapy with 40 fractions of $120 \mathrm{cGy}$ twice daily, resulting in a total dose of $4800 \mathrm{cGy}$. An incisional wound was made 4 weeks post radiotherapy to allow time for completion of the inflammatory and proliferative processes before onset of the maturation phase of tissue repair. Wound repair was assessed microscopically. Of the rats in the control group, $16 \%$ exhibited partial epithelisation and granulation tissue, $32 \%$ exhibited fibrosis, and $28 \%$ presented with mild or severe inflammation.

By contrast, in the conventional group, 13\% exhibited partial epithelisation and granulation, 35\% exhibited fibrosis, and 19\% presented with mild or severe inflammation. These results imply that there was no significant difference in tensile strength between the control and the conventional groups $(\mathrm{p}=0.07)$. Of the rats in the hyperfractionated group, $12 \%$ exhibited partial epithelialisation, 
$18 \%$ exhibited granulation tissue, $62 \%$ exhibited fibrosis, and $31 \%$ presented with mild or severe inflammation. The results of a histopathologic analysis indicated that the occurrence of fibrosis increased significantly $(p=0.038)$ in the hyper-fractionated group in contrast to the other groups, and the rats in the hyper-fractionated group had significantly lower wound tensile strength than those in the control group ( $p=0.03 ; 95 \%$ CI: 20.9647-42.6603). Although research using animal models has added considerably to our scientific knowledge, controversy remains as to how this knowledge translates to human wound healing given that rats heal through contraction, whereas humans heal through re-epithelisation..$^{28}$ It is important to acknowledge the anatomical distinctions between animal models and humans, in addition to acknowledging the differences in physiological responses and pathological processes. $^{28}$

\section{PREDICTORS OF WOUND COMPLICATIONS}

A review by Brown and Rzucidlo discussed the patient factors associated with an increased risk of the tissue effects of radiotherapy. ${ }^{29}$ Based on a review of a series of case reports, the authors suggested an increased risk of wound complications in patients diagnosed with connective tissue disorders, such as lupus or scleroderma. Other case reports have linked patients with ataxia telangiectasia (a rare autosomal disorder) to an increased risk of the tissue effects of radiotherapy. In addition to other cellular DNA repair abnormalities or chromosomal breakage syndromes, obesity has been shown to be a factor elevating the risk of tissue effects because of the higher doses of radiotherapy required to penetrate through the body. Finally, chemotherapeutic agents, which are used to sensitise patients to radiotherapy or as an adjunct to treatment, were found to increase an individual's susceptibility to the tissue effects of radiotherapy. Case reports add to the body of evidence by presenting the findings of clinicians in practice, but the value of such reports remains controversial because they are low on the hierarchy of evidence. ${ }^{30}$

LeBrun et al. undertook a retrospective, observational cohort study of 67 patients who underwent soft tissue sarcoma resection and radiotherapy. ${ }^{31}$ The primary outcome was the occurrence of any wound complication up to 4 months postoperatively. The authors found that $12 \%$ (n $=8$ ) of the patients had minor wound complications and $21 \%(\mathrm{n}=14)$ had major wound complications documented within a median of 30 days postoperatively. In addition, diabetes was found to be associated with an increased risk of the development of major wound complications (OR 5.10; 95\% CI 1.07-24.29; $\mathrm{p}=0.4$ ). Other predictors of wound complications included radiation dermatitis (grade 2 or above) (OR 4.82; 95\% CI 1.20-19.21; $\mathrm{p}=0.03$ ). Interestingly, the patients who underwent proton therapy did not have any wound complications. This finding may be ascribed to the increased precision of proton therapy compared to that of conventional radiotherapy, which minimises the dose to normal tissue. 1 In comparison, the patients treated with three-dimensional (3D) conformal radiotherapy suffered from more wound complications compared to those treated with intensity-modulated radiotherapy (IMRT) $(p=0.04)$. Moreover, the authors found no statistically significant link between the patients who received radiotherapy preoperatively and those who received it postoperatively $(\mathrm{p}>.05)$. By contrast, in a more recent study, Stevenson et al. found a correlation between preoperative external beam radiotherapy and major wound complications (OR 2.75; 95\% CI 1.21-6.26; P = 0.02). ${ }^{32}$

According to the results of a study by LeBrun et al., proton therapy and IMRT were associated with a reduced risk of wound complications in comparison to $3 \mathrm{D}$ conformal radiotherapy. ${ }^{31}$ However, a limitation of their study is their reliance on the accuracy of retrospective information because data may be absent or of poor quality. 33 Furthermore, the follow-up time of 4 months was short. True effects of any medical treatment may not be known for some time $e^{34}$ therefore this is a potential limitation of the study.

\section{CONCLUSION}

Radiotherapy has a multifaceted cellular effect on tissue repair, involving cytokines, growth factors, fibroblasts, ECM, MMPs, and collagen synthesis, although the pathogenesis is not fully understood yet. ${ }^{3-4}$ Fractionated radiotherapy treatment induces a repetitive cycle of inflammation. ${ }^{3}$ The acute effects of radiotherapy are mostly transient and are resolved within 4 weeks of the last dose, which correlates with the normal pathogenesis of skin regeneration. ${ }^{3-4}$

Two main theories pertaining to the late effects of radiotherapy can be found in the literature. Marx proposed that a chronic wound is caused by the hypoxic-hypocellularhypovascular effects of treatment. ${ }^{17}$ Dion et al. argued that damage occurs because of changes in microvasculature, in addition to the depletion of parenchymal and stromal cells. $^{20}$ Ozbek et al. found that radiotherapy reduces the tensile strength of wounds. ${ }^{27}$ This finding could explain why a few individuals who have undergone radiotherapy present with a wound following minimal trauma. More recently LeBrun et al. proposed that the treatment modality used, for example, proton therapy and IMRT, can influence patient outcomes in relation to tissue injury. ${ }^{31}$

Advancement in our understanding of radiotherapy and its effects may lead to improvement in the type of treatment modalities available, thereby preventing tissue injury altogether. It is imperative that clinicians have knowledge 
and understanding of the pathophysiology of the intrinsic and extrinsic factors that influence wound healing. This will enable clinicians to provide optimal and realistic treatment therapies to patients.

\section{IMPLICATIONS FOR CLINICAL PRACTICE}

Clinicians need to appreciate that healing of a radiation wound may not be realistic.

Symptom-relieving treatment is of paramount importance to address the deleterious consequences of a radiotherapy related wound.

Clinicians need to be cognisant of the need to maintain the patient's self-esteem and optimise their quality of life.

\section{FURTHER RESEARCH}

Studies examining the combined effect of pentoxyifylline and vitamin to reduce the side effects of radiotherapy are ongoing and have produced contrasting findings. Therefore, more evidence is needed to determine whether this should be implemented.

\section{REFERENCES}

1. Gieringer M, Gosepath J, Naim R. Radiotherapy and wound healing: principles, management and prospects. Oncology Reports. 2011 Aug 1;26(2):299 307

2. Gianfaldoni S, Gianfaldoni R, Wollina U, Lotti J, Tchernev G, Lotti T. An overview on radiotherapy: from its history to its current applications in dermatology. Open Access Macedonian Journal of Medical Sciences. 2017 Jul 25;5(4):521.

3. Jacobson LK, Johnson MB, Dedhia RD, Niknam-Bienia $S$, Wong AK. Impaired wound healing after radiation therapy: A systematic review of pathogenesis and treatment. JPRAS Open. 2017 Sep 1;13:92-105.

4. Olascoaga A, Vilar-Compte D, Poitevin-Chacón A, Contreras-Ruiz J. Wound healing in radiated skin: pathophysiology and treatment options. International Wound Journal. 2008 May; 5(2):246-57.

5. Haubner F, Ohmann E, Pohl F, Strutz J, Gassner HG. Wound healing after radiation therapy: review of the literature. Radiation Oncology. 2012 Dec;7(1):162.

6. Glover D, Harmer V. Radiotherapy-induced skin reactions: assessment and management. British Journal of Nursing. 2014 Feb;23(Sup2):S28-35.

7. Enoch $S$, Leaper DJ. Basic science of wound healing. Surgery (Oxford). 2008 Feb 1;26(2):31-7.

8. Young A, McNaught CE. The physiology of wound healing. Surgery (Oxford). 2011 Oct 1;29(10):475-9. Flanagan $M$. Wound healing and skin integrity: principles and practice. John Wiley \& Sons; 2013 Feb 26.

9. De Almeida SM, Cruz AD, Ferreira RI, Vizioli MR, Bóscolo FN. Effect of low-dose electron radiation on rat skin wound healing. Brazilian Dental Journal. 2007; 18(3):208-14.

10. McQuestion M. Evidence-based skin care management in radiation therapy: clinical update. In Seminars in Oncology Nursing 2011 May 1 (Vol. 27 No. 2, pp. e1-e17). WB Saunders.

11. Denham JW, Hauer-Jensen M. The radiotherapeutic injury-a complex 'wound'. Radiother Oncol. 2002 May 1;63(2):129-45.

12. Ryan JL. lonizing radiation: the good, the bad, and the ugly. J Invest Dermatol. 2012 Mar 1;132(3):985-93.
13. Dormand EL, Banwell PE, Goodacre TE. Radiotherapy and Wound Healing. Int Wound J. 2005 Jun 2 (2): 112-27

14. Liu X, Liu JZ, Zhang E, Li P, Zhou P, Cheng TM, et al. Impaired wound healing after local soft $X$-ray irradiation in rat skin: time course study of pathology, proliferation, cell cycle, and apoptosis. Journal of Trauma and Acute Care Surgery. 2005 Sep 1;59(3):682-90.

15. Keskikuru R, Jukkola A, Nuutinen J, Kataja V, Risteli J, Autio $P$, et al. Radiation-induced changes in skin type I and III collagen synthesis during and after conventionally fractionated radiotherapy. Radiother and Oncol. 2004 Mar 1:70(3):243-48.

16. Marx RE. A new concept in the treatment of osteoradionecrosis. J Oral Maxillofac Surg. 1983 Jun 1;41(6):351-57.

17. Nadella KR, Kodali RM, Guttikonda LK, Jonnalagadda A. Osteoradionecrosis of the jaws: clinico-therapeutic management: a literature review and update. J Maxillofac Oral Surg. 2015 Dec 1;14(4):891-901.

18. Devalia HL, Mansfield L. Radiotherapy and wound healing. International Wound Journal. 2008 Mar;5(1):40-4

19. Dion MW, Hussey DH, Doornbos JF, Vigliotti AP, Wen $\mathrm{BC}$, Anderson B. Preliminary results of a pilot study of pentoxifylline in the treatment of late radiation soft tissue necrosis. International Journal of Radiation Oncology*Biology*Physics. 1990 Aug 1;19(2):401-7.

20. Pareek P, Samdariya S, Sharma A, Gupta N, Shekhar S, Kirubakaran R. Pentoxifylline and vitamin E alone or in combination for preventing and treating side effects of radiation therapy and concomitant chemoradiotherapy. The Cochrane Database of Systematic Reviews. 2016 Mar;2016(3).

21. Famoso JM, Laughlin B, McBride A, Gonzalez VJ. Pentoxifylline and vitamin $E$ drug compliance after adjuvant breast radiation therapy. Advances in Radiation Oncology. 2018 Jan 1;3(1):19-24.

22. Illsley MC, Peacock JH, McAnulty RJ, Yarnold JR. Increased collagen production in fibroblasts cultured from irradiated skin and effect of TGF 1-clinical study. British Journal of Cancer. 2000 Sep;83(5):65054.
23. Goessler UR, Bugert $P$, Kassner $S$, Stern-Straeter J, Bran $G$, Sadick $H$, et al. In vitro analysis of radiationinduced dermal wounds. Otolaryngology-Head and Neck Surgery. 2010 Jun; 142(6):845-50.

24. Broughton G II, Janis JE, Attinger CE. Wound healing: an overview. Plast Reconstr Surg. 2006 Jun 1;117(7S): 1 e-S.

25. Schaffer $M$, Weimer W, Wider $S$, Stülten $C$, Bongartz M, Budach M, Becker HD. Differential expression of inflammatory mediators in radiation-impaired wound healing. J Surg Res. 2002 Sep; 107(1):93-100.

26. Ozbek N, Guneren E, Yildiz L, Meydan D, Cakir S, Coskun $M$. The effect of pre-operative conventional and hyperfractionated radiotherapy schedules on wound healing and tensile strength in rats: an experimental study. Int J Oral Maxillofac Surg. 2005 Mar 1;34(2):185-92.

27. Nuutila K, Katayama S, Vuola J, Kankuri E. Human wound-healing research: issues and perspectives for studies using wide-scale analytic platforms. Advances in Wound Care. 2014 Mar 1;3(3):264-71.

28. Brown, K, R. Rzucidlo, E. Acute and chronic radiation injury. J Vasc Surg. 2011 Jan;53 (1):15S-21S.

29. Greenhalgh, T. How to read a paper; the basics of evidence-based medicine. 5th ed. Chichester: John Wiley \& Sons Ltd; 2014.

30. LeBrun DG, Guttmann DM, Shabason JE, Levin WP, Kovach SJ, Weber KL. Predictors of wound complications following radiation and surgical resection of soft tissue sarcomas. Sarcoma. 2017;2017:Article ID 5465130.

31. Stevenson MG, Ubbels JF, Slump J, Huijing MA Bastiaannet $E$, Pras $E$, et al. Identification of predictors for wound complications following preoperative or postoperative radiotherapy in extremity soft tissue sarcoma. Eur J Surg Oncol. 2018 Jun 1;44(6):816-22.

32. Sedgwick P. Retrospective cohort studies: advantages and disadvantages. BMJ. 2014 Jan 24;348:g1072

33. Evans I, Thornton H, Chalmers I, Glasziou P. Testing treatments: better research for better healthcare. Pinter \& Martin Publishers; 2011 